\title{
List of Illustrations and Tables
}

\section{ILLUSTRATIONS}

1.1. Michael Faraday's 1821 electric motor 2

1.2. Laboratory blueprint (1979) 6

1.3. Peter Barlow's 1821 star electric motor 9

1.4. Thomas Davenport's 1837 electric motor II

2.1. Joseph Wright of Derby, The Orrery (c. 1764) 22

2.2. John Rowley's orrery (c. 1713) 26

2.3. James Ferguson's solar moon orbit orrery (1809) 27

2.4. John Smeaton's model waterwheel (1809) 30

2.5. James Watson, Francis Crick, and their DNA model (1953) 35

3.1. Jules Salleron's pulse glass (1864) 42

3.2. Joseph Wright of Derby, Experiment with the Airpump (1768)

3.3. Cutaway diagram of a cyclotron (1990) 50

3.4. Top schematic view diagram of a cyclotron (1990) 51

3.5. Cyclotron resonance graph (1931) 53

3.6. MIT cyclotron (c. 1960) 55

3.7. Cyclotron electrostatic focusing (1990) 57

3.8. Cyclotron weak magnetic focusing (1990) 58

3.9. Wilson seal for the cyclotron (1990) 60

3.10. Mechanism for converting linear to rotary motion across a vacuum seal (1990) 
4.I. Diffraction schematic (1991) 72

4.2. Rowland circle (1991) 73

4.3. Spectral analysis working curve (1948) 76

4.4. Schematic diagram of a photomultiplier tube (1947) 77

4.5. Schematic diagram of a direct-reading spectrometer (1947) $\quad 78$

4.6. Direct-reading spectrometer optics diagram (1945) 82

4.7. Photomultiplier tube sensitivity graph (1945) 83

7.1. Henry Rowland and his ruling engine (c. 1890) 150

7.2. Rowland's ruling engine blueprint (1902) 151

7.3. Spectrometry room (c. 1940) 153

7.4. Delivery of early Baird Associates spectrograph (c. 1940) 154

7.5. Baird-Atomic photomultiplier tube rack (c. 1960) 158

7.6. Drawing of Baird Associates three-meter spectrograph (c. 1942)

160

7.7. Diagram of a split slit (2000) 161

$\begin{array}{ll}\text { 7.8. Scan of a Kleenex (1999) } & 167\end{array}$

8.1. Thompson's improved indicator (c. 1870) 172

8.2. Émile Clapeyron's pressure-volume graph (1834) 173

8.3. Expansive working (1782) 175

8.4. Graph from indicator diagram (c. 1803) 185

9.1. Baird Associates direct-reading spectrometer advertisement, $\begin{array}{ll}\text { inside (c. 1954) } & 202\end{array}$

9.2. Baird Associates direct-reading spectrometer advertisement, front page (c. 1954) 204

10.1. Magnetic resonance image, Gibbs ringing artifact (1988) $\quad 216$

10.2. Magnetic resonance image, Gibbs ringing artifact amelioration (1988)

10.3. Magnetic resonance image, classic v. standard MR images (1999)

10.4. Magnetic resonance image, intervertebral spinal disc bulges (1999)

10.5. Baird Associates three-meter spectrometer advertisement (c. 1938 ) 
TABLES

6.1. Spectrometer/Spectrograph Calibration 126

10.1. Baird Associates Balance Sheet, 1936-1946 
This page intentionally left blank 